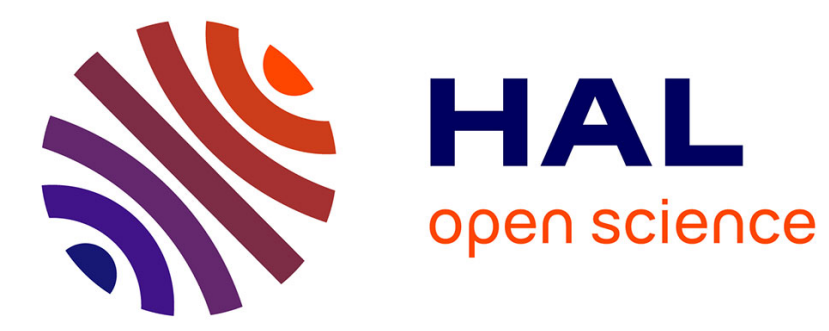

\title{
Ion velocity distribution function observations in a multipolar argon discharge
}

G Bachet, Laurence Chérigier-Kovacic, Fabrice Doveil

\section{To cite this version:}

G Bachet, Laurence Chérigier-Kovacic, Fabrice Doveil. Ion velocity distribution function observations in a multipolar argon discharge. Physics of Plasmas, 1995, 2 (5), pp.1782-1788. 10.1063/1.871328 . hal-03534517

\section{HAL Id: hal-03534517 https://hal.science/hal-03534517}

Submitted on 19 Jan 2022

HAL is a multi-disciplinary open access archive for the deposit and dissemination of scientific research documents, whether they are published or not. The documents may come from teaching and research institutions in France or abroad, or from public or private research centers.
L'archive ouverte pluridisciplinaire HAL, est destinée au dépôt et à la diffusion de documents scientifiques de niveau recherche, publiés ou non, émanant des établissements d'enseignement et de recherche français ou étrangers, des laboratoires publics ou privés. 


\section{AIP $\left.\right|_{\text {Physics of }}$

Ion velocity distribution function observations in a multipolar argon discharge

G. Bachet, L. Chérigier, and F. Doveil

Citation: Physics of Plasmas 2, 1782 (1995); doi: 10.1063/1.871328

View online: $h$ ttp://dx.doi.org/10.1063/1.871328

View Table of Contents: http://scitation.aip.org/content/aip/journal/pop/2/5?ver=pdfcov

Published by the AIP Publishing

\section{Articles you may be interested in}

Diagnosis of ion velocity distribution from pin to plate geometry in atmospheric argon dielectric barrier discharge

Phys. Plasmas 20, 073302 (2013); 10.1063/1.4816024

Ion acceleration in $\mathrm{Ar}-\mathrm{Xe}$ and $\mathrm{Ar}-\mathrm{He}$ plasmas. II. Ion velocity distribution functions

Phys. Plasmas 17, 113509 (2010); 10.1063/1.3505823

Reconstruction of ion energy distribution function in a capacitive rf discharge

Appl. Phys. Lett. 94, 211503 (2009); 10.1063/1.3147216

Laser-induced-fluorescence observation of ion velocity distribution functions in a plasma sheath

Phys. Plasmas 13, 062103 (2006); 10.1063/1.2206786

The ion velocity distribution function in a current-free double layer

Phys. Plasmas 12, 093502 (2005); 10.1063/1.2033647

\section{Did your publisher get}

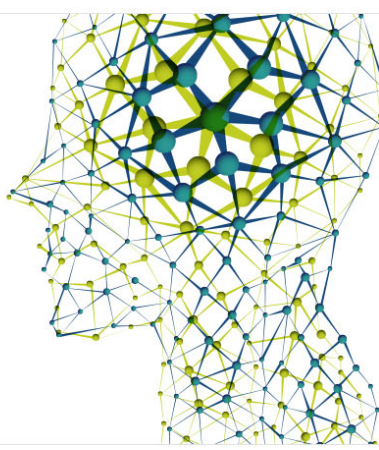




\title{
Ion velocity distribution function observations in a multipolar argon discharge
}

\author{
G. Bachet, L. Chérigier, and F. Doveil \\ Equipe Turbulence Plasma, URA 773 du CNRS et de l'Université de Provence, Institut Méditerranéen de \\ Technologie, F-1345I Marseille cedex 20. France
}

(Received 26 October 1994; accepted 21 December 1994)

\begin{abstract}
Observations of ion velocity distribution functions in two different configurations of a multipolar device with a laser induced fluorescence diagnostic in argon plasma are presented. The first experiment, carried out in the magnetic field closing the two ends of the device, shows the influence of this field on the ion trajectories. In the second experiment performed without the magnetic field closing the two ends of the device, the influence of the electrostatic boundary potential of the plasma is observed. Longitudinal ion velocity distribution functions have been recorded until inside the plasma sheath. The shapes of these distribution functions are in qualitative agreement with the results of a theoretical model and give an experimental justification for the "flux" shape of the ion velocity distribution source function retained in this model. However, to reach this qualitative agreement, it is necessary to adjust the ionic temperature for each distribution function suggesting that, for the authors' weakly ionized plasma, another heating mechanism is missing in this theory. () 1995 American Institute of Physics.
\end{abstract}

\section{INTRODUCTION}

As is well known, laser induced fluorescence ${ }^{1-4}$ is a nonintrusive technique based on the Doppler effect, which gives a very accurate measurement of the velocity profile of the ion distribution function in a plasma. In order to investigate the regime of a steady state argon plasma at low gas pressure, we have developed a laser induced fluorescence diagnostic ${ }^{5.6}$ using a continuous wave 899-21 ring dye laser from Coherent to pump the AII transition $3 d^{2} G_{9 / 2}-4 p^{2} F_{7 / 2}$, the fluorescence being observed on the $4 p^{2} F_{7 / 2}-3 s^{2} D_{5 / 2}$ transition.

The plasma is created by a thermoelectronic discharge in a multicusp magnetic field device. It has already been showed that such devices produce a large volume of unmagnetized plasma ${ }^{7.8}$ and serve the purpose of basic experiments. In our device, a typical argon plasma is obtained by a $1 \mathrm{~A}, 60$ $V$ discharge between a 4 filaments holder and the walls of the vacuum vessel in a range pressure of $10^{-4}-610^{-4}$ Torr. The plasma density can be varied from $10^{8}$ to $510^{9} \mathrm{~cm}^{-3}$.

The cylindrical vessel is specially designed to do both longitudinal and radial optical exploration of the plasma at any point along the axis of the device. We choose to mainly concentrate on the role of the longitudinal boundary of the plasma which can be either the magnetic multipolar field or the electrostatic field of the wall-plasma sheath. Beside being a perturbative technique, Langmuir probe measurements in a magnetized region or in electrostatic sheaths can be questioned and absolute measurements are always difficult. Whereas laser induced fluorescence appears to be an interesting tool for this study.

This paper is organized as follows. In Sec. II we present results obtained in presence of the magnetic field at the two end doors of the device. Previous work has shown the trapping of primary electrons in the magnetic cusps ${ }^{9.10}$ and the plasma diffusion through a multipolar magnetic field. ${ }^{11,12} \mathrm{We}$ give another insight on the ion diffusion toward the magnetic cusps by measuring the ion drift velocity. In Sec. III we present experiments carried out without this ending magnetic field and study the shape of the ion velocity distribution functions in the neighborhood and inside the electrical sheath at the wall. The principal assumptions of Emmert et al.'s theory ${ }^{13}$ allowing the analytical calculation of the ion velocity distribution function at any point of the plasma are recalled and our results are confronted to these predictions. In Sec. IV we state our conclusions.

\section{MULTIPOLAR MAGNETIC FIELD EFFECTS ON ION TRAJECTORIES}

Four rows of permanent magnets with alternating polarity and equally spaced are held by each end door of the device. As shown on Fig. 1(a), one side of the rows is in a plane located at the edge of the rectangular optical windows used to beam the laser transversally through the plasma and to collect the fluorescence. The doors can rotate around the cylinder axis allowing the transversal laser beam to be either parallel or perpendicular to the magnets. Taking advantage of this fact. we have realized two series of experiments to avoid any ambiguity on the ion flux directions. First, the magnet rows are parallel to the transversal laser beam along the $y$ axis (the coordinates refer to the magnet rows on Fig. 1). We locally measure the velocity distribution function at several points along the cylinder axis and thus we obtain the mean velocity component $v_{y}$. Second, the magnets are perpendicular to the transversal laser beam. The mean velocity component $v_{z}$ is then obtained. Finally, we can also beam the laser along the cylinder axis $x$ through two small portholes on both ends of the device. This allows the measurement of the longitudinal ion velocity distribution function. We verified that the longitudinal velocity component $v_{x}$ obviously did not depend on the orientation of the magnet rows with respect to the laser beam direction. In Fig. 2 these mean velocities are plotted versus the distance to the magnet plane.

We can distinguish between two regions in the device. In 


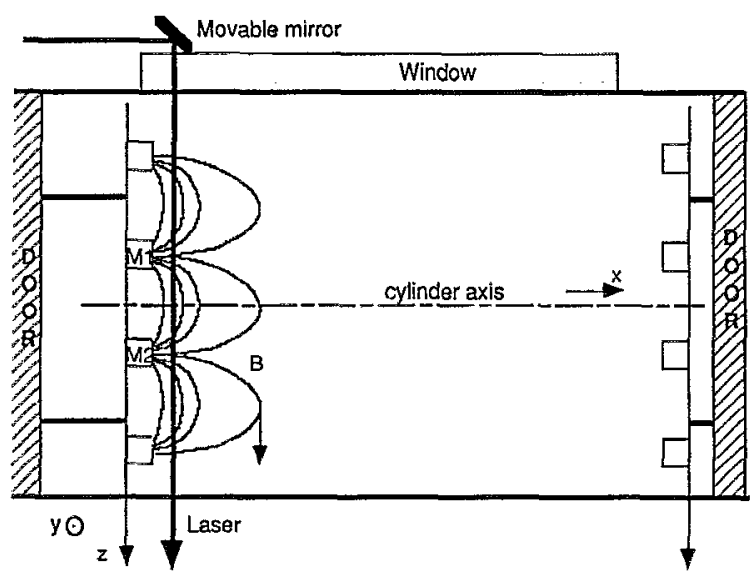

(a)

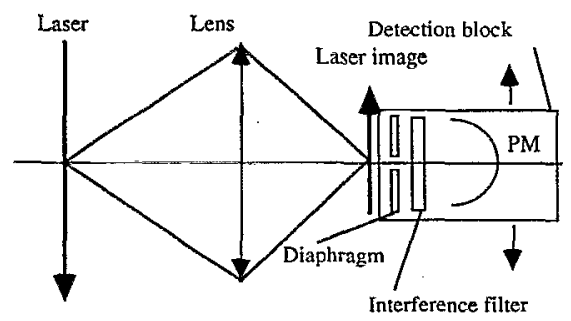

(b)

FIG. 1. Experimental setup. (a) View of the multipole geometry for the measurement of velocity distribution function in the magnetic field. (b) Schematic diagram of the fluorescence detection system.

the central region where the plasma is unmagnetized, transversal velocities $v_{y}$ and $v_{z}$ are equal to zero on the axis. Only the absolute value of the velocity in the direction of the axis of the device $\left(v_{x}\right)$ increases because of the electric field when measuring closer to the limit of the magnetized region. When the ions enter this second region, which extends about $10 \mathrm{~cm}$ from the magnets, the trajectories of the ions are modified by the magnetic field. Moreover, as shown by the

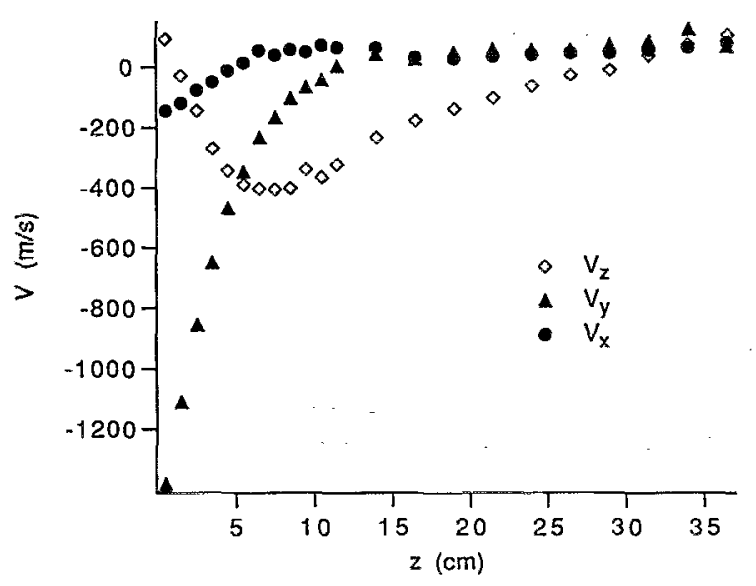

FIG. 2. Mean velocity components versus the distance to the plane containing the magnets.

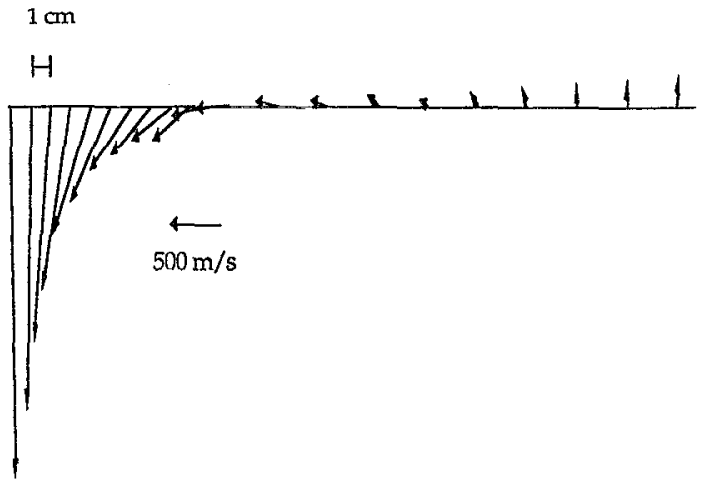

FIG. 3. Velocity vectors in the $x-y$ plane.

large values of $v_{y}$ observed on Fig. 2, the transversal ion velocity distribution functions measured at the top of one magnetic arch clearly exhibit an important ion flux in the direction parallel to the magnets. Because of the presence of the magnetic field $B$ and of an electric field $E$ due to the boundary leaks, this flux can obviously be attributed to the resulting $E \times B$ drift. On Fig. 3 the projection of the velocity vector in the $x-y$ plane (containing the device axis) is presented. It clearly shows that ions are moving from the center of the device toward each door and accelerated by the potential gradient which can be measured with a Langmuir probe. ${ }^{5}$ We have also performed a simple numerical ion trajectory calculation and shown that weak electric fields $(<1 \mathrm{~V} / \mathrm{m})$ can lead to such an acceleration. Figure 3 also displays the $E \times B$ ion drift in the nonzero magnetic field region. For symmetry reasons, this drift only exists at the top of the magnetic arches.

In order to complete this study, we recorded the distribution functions very close $(5 \mathrm{~mm})$ to the plane containing the magnets along a line following the transversal laser beam perpendicular to the magnets. This can be done by moving the detection block of the fluorescence (diaphragm, optical interferential filter, photomultiplier) in the plane containing the laser beam image given by the collecting lens [Fig. 1(b)]. The mean velocity of each distribution function is reported in Fig. 4(a) for several points between two magnets. Because of technical reasons, the laser beam is not exactly perpendicular to the magnet rows. This induces a slight negative shift in the velocity components (which must be also taken in account for the values of $v_{z}$ on Fig. 2 in the magnetized region). We observe that ions are moving from the center of the magnetic arch toward the magnets. The full width at half-maximum of the measured distribution functions also gives a determination of the ion temperature along the laser beam. Figure 4(b) shows an increase of the temperature along $z$ when the cusps are reached. A similar increase can be observed on the longitudinal data along the cylinder axis, with the longitudinal temperature varying from an average value of $0.04 \mathrm{eV}$ in the nonmagnetized region to $0.14 \mathrm{eV}$ between two magnets. We first thought that this increase in the ion temperature was due to the Zeeman effect. But measurements of the magnetic field showed that it is not strong enough to induce the nec- 


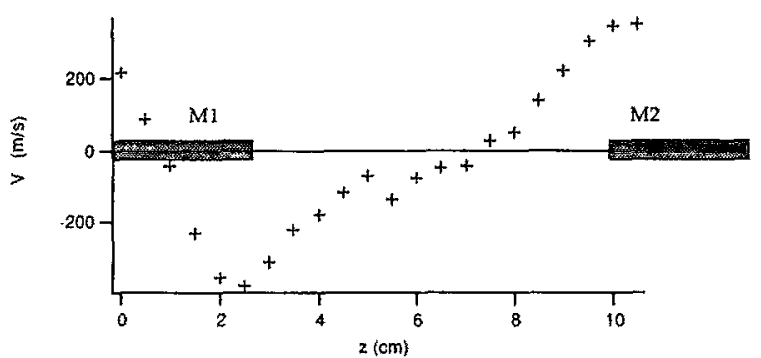

(a)

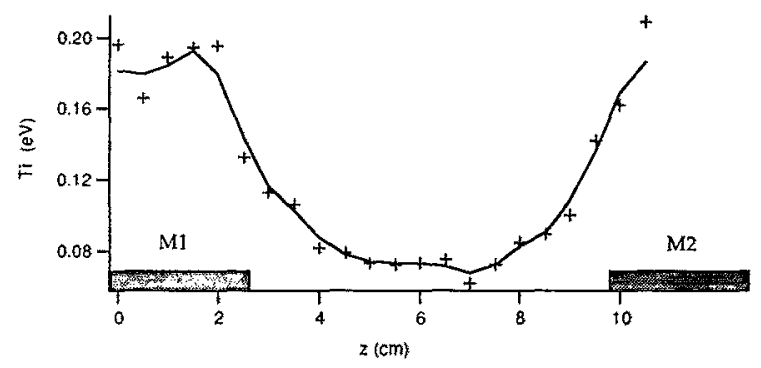

(b)

FIG. 4. (a) Mean velocity component $v_{z}$ vs the distance to the center of magnet M1. (b) Ion perpendicular temperature versus the distance to the center of magnet $\mathrm{M} 1$.

essary Zeeman effect. Moreover, by taking the direction of linear polarization of the laser beam perpendicular to the previous one, the same results are obtained: In neither case the two $\sigma$ Zeeman components can be resolved making sure that the Zeeman effect is too small to explain this heating. It is worth being noticed that the magnetic moment $\mu$ associated to the motion of a charged particle in the magnetic field and defined by

$$
\mu \equiv \frac{1}{2} m \nu_{\perp}^{2} / B \equiv K T_{i \perp} / B
$$

appears to be conserved in the magnetic field region within about $6 \mathrm{~cm}$ from the magnets. The observed temperature increase with the magnetic field $B$ can thus be related to the conservation of this adiabatic invariant, although the magnetic field is rapidly varying.

\section{DISTRIBUTION FUNCTION SHAPE WITHOUT MAGNETIC FIELD ENDING}

To see the influence of the plasma sheath on the ion distribution function we suppress the magnets held by the two end doors of the device and replace them by ending plates which can be grounded or held electrically floating. Measurements can thus be done until inside of the electrostatic sheath bounding the plasma.

The study of the sheath potential has motivated many analytical $^{13-21}$ and numerical ${ }^{22-25}$ works. One of these analytical works is due to Emmert et al. ${ }^{13}$ whose calculated ion velocity distribution functions (Fig. 6 of Ref. 13) seem, at

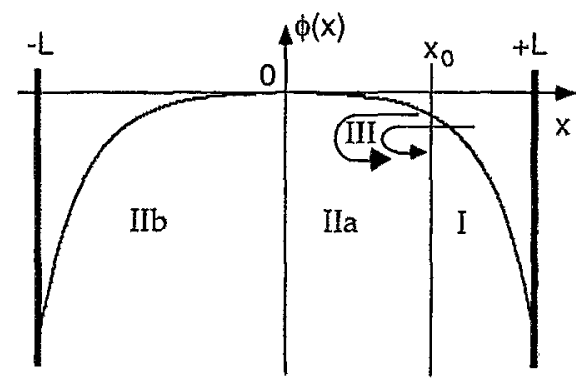

(a)

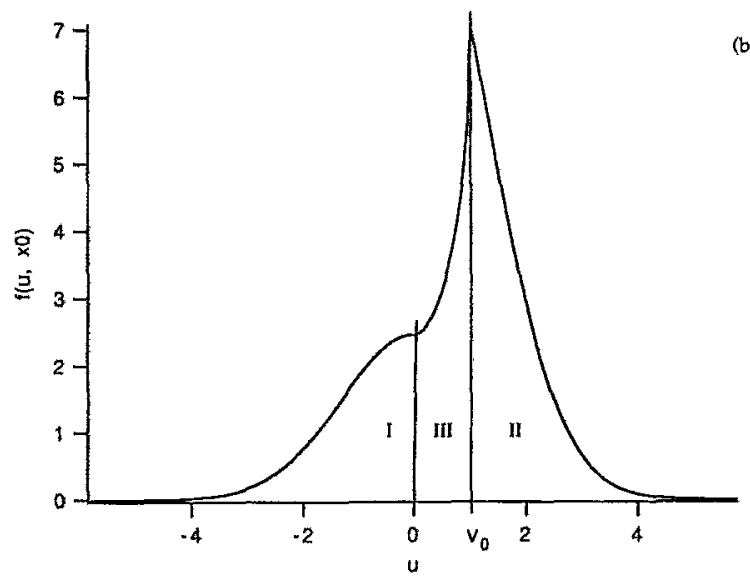

FIG. 5. (a) Geometry of Emmert et al.'s one dimensional model. (b) Theoretical ion distribution function at $x_{0}$.

first sight, qualitatively similar to our recorded longitudinal distribution functions. The experimental transversal distribution functions are Maxwellian as in the assumptions of Emmert's model, ${ }^{13}$ so that we can use this one dimensional model to explain our results.

This model is based on a kinetic description of the ions, the electrons following the Boltzmann relation

$$
n_{e}(x)=n_{e 0} \exp \left(\frac{e \phi(x)}{k T_{e}}\right) \text {. }
$$

The total energy for the ions is

$$
E=\frac{1}{2} m_{i} \nu_{i}^{2}+q_{i} \phi(x) \text {, }
$$

where $q_{i}=Z e$ is the ion charge. The ion energy is a constant of motion and for the steady-state solution, the kinetic equation reduces to

$$
\frac{d}{d x}\left[g_{i}(x, E) \nu_{i}(x, E)\right]=S_{i}(x, E),
$$

where $S_{i}(x, E)$ is the "ion source function" and $g_{i}(x, E)$ the ion energy distribution function. The ion density can be easily obtained

$$
n_{i}(x)=\sum_{R} \int_{q_{i} \phi(x)}^{\infty} \int_{0}^{L} \frac{S_{i}\left(x^{\prime}, E\right)}{\nu_{i}(x, E)} d x^{\prime} d E,
$$

where the sum is over the various regions $R$ [Fig. 5(a)] in the 


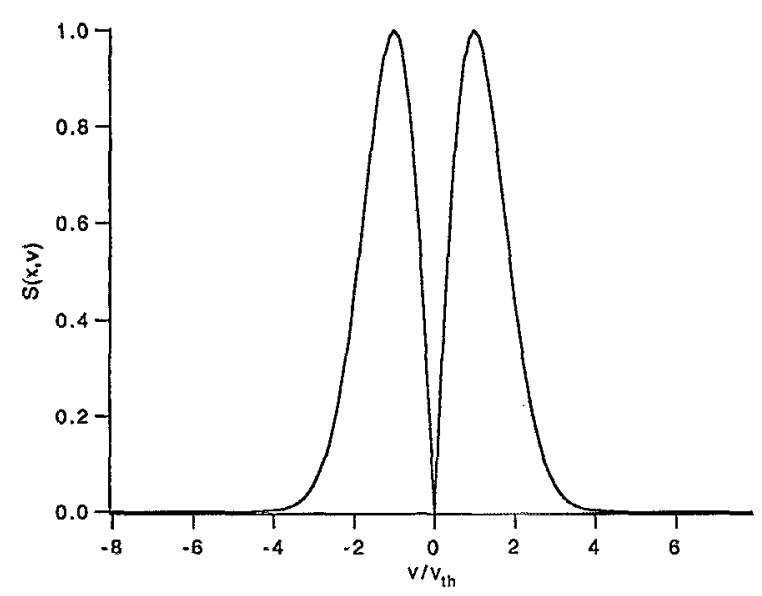

FIG. 6. Flux type source function.

system which contributes to the ion density at $x$. Inserting Eqs. (1) and (4) into the Poisson's equation, the integrodifferential plasma-sheath relation is obtained

$$
\begin{aligned}
\frac{d^{2} \phi(x)}{d x^{2}}= & -4 \pi e\left[\sum_{R} \int_{q_{i} \phi(x)}^{\infty} \int_{0}^{L} \frac{S_{i}(x, E)}{\nu_{i}(x, E)} d x^{\prime} d E\right. \\
& \left.-n_{e 0} \exp \left(\frac{e \phi(x)}{k T_{e}}\right)\right] .
\end{aligned}
$$

If one assume that the region outside the sheath is quasineutral, which means

$$
\frac{d^{2} \phi(x)}{d x^{2}}=0,
$$

Eq. (5) can be analytically integrated. The energy distribution source function used by Emmert et al. ${ }^{13}$ is

$$
S_{i}(x, E)=\frac{S_{0 i} h(x)}{k T_{i}} \exp \left(\frac{-E}{k T_{i}}\right) \exp \left(\frac{q_{i} \phi(x)}{k T_{i}}\right),
$$

where the factor $h(x)$ is the "spatial ion source" intensity profile. This energy source function yields, like pointed out by Procassini et al., ${ }^{22}$ to a velocity distribution source function of the "flux" type

$$
S_{i}\left(x, \nu_{x}\right)=\frac{S_{0 i} h(x) m_{i} \nu_{x}}{k T_{i}} \exp \left(\frac{-m_{i} \nu_{x}^{2}}{2 k T_{i}}\right) .
$$

This equation means that there is no ion created with a zero velocity as shown on Fig. 6 . With these assumptions, the electrostatic potential can be obtained and the ion velocity distribution function in any place of the one-dimensional plasma is given by

$$
f\left(x, \nu_{x}\right)=H \frac{n_{0}}{Z}\left(\frac{m_{i}}{2 \pi k T_{i}}\right)^{1 / 2} \exp \left(\frac{Z T_{e} \Psi_{0}}{T_{i}}\right),
$$

where

$$
\Psi_{0}=\Psi(0)=\Psi-\frac{m_{i} \nu_{x}^{2}}{2 k T_{e} Z},
$$

$$
\begin{aligned}
& H=1+F(\Psi) \text { for } \nu_{0}<\nu_{x}, \\
& H=1+F(\Psi)-2 F\left(\Psi_{0}\right) \text { for } 0<\nu_{x}<\nu_{0}, \\
& H=1-F(\Psi) \text { for } \nu_{x}<0, \\
& \nu_{0}=\left[\frac{2 Z k T_{e} \Psi}{m_{i}}\right]^{1 / 2},
\end{aligned}
$$

and

$$
\begin{aligned}
F(\Psi)= & \operatorname{erf}\left[\left(Z \frac{T_{e}}{T_{i}} \Psi\right)^{1 / 2}\right]+\frac{2 D(\sqrt{\Psi})}{\left[\pi Z\left(T_{e} / T_{i}\right)\right]^{1 / 2}} \\
& \times \exp \left[-\left(1+Z \frac{T_{e}}{T_{i}}\right) \Psi\right]
\end{aligned}
$$

$\operatorname{erf}(z)$ is the error function, $D(z)$, the Dawson function and $\psi=(-e \phi) /\left(k T_{e}\right)$.

From Eq. (11) we can see that the distribution function at a given point $x_{0}$ consists into three parts [Fig. 5(b)]:

- a left part which is half a Gaussian distribution centered on zero velocity [Fig. 5(b) -region I] due to the ions created in region $I$ of Fig. 5(a) with a negative velocity;

-a right part which is also a part of a Gaussian distribution centered on zero velocity but truncated at $v_{0}$ [Fig. 5(b) -region II], where $v_{0}$ is defined in Eq. (12) and is the velocity of an ion initially at rest on top of the potential hill and accelerated to $x_{0}$. This part of the distribution function is coming from the acceleration by the electrostatic field of ions created in region IIa with a positive velocity and of the ions created in region IIb having enough energy to climb the potential well;

-and a middle part [Fig. 5(b)-region III] which connects the two others and which is made of the ions created in I and IIa with a negative velocity but reflected by the potential well in region IIa and ions created in IIa with a velocity less than $v_{0}$ even after being accelerated by the potential gradient.

The respective magnitude of the two first contributions is related to the size of regions I and II with $x_{0}$.

In Figs. 7, 8, and 9 we present our experimental results recorded at several locations along the $x$ axis. Figures 7 and 8 show the distribution functions when the plate is electrically floating, as in Emmert et al.'s model, ${ }^{13}$ for an argon pressure of $6 \times 10^{-4}$ and $10^{-4}$ Torr, respectively; while in the case of Fig. 9, the plate is grounded and the pressure is $10^{-4}$ Torr. We used Emmert et al.'s model ${ }^{13}$ to fit numerically the experimental curves. The adjustable parameters are the amplitude, the electron, and ion temperatures and the potential. The latter is related to the experimental value of $v_{0}$. The electron temperature is also taken as the value given by a probe measurement since we noticed that the electron temperature does not influence much the shape of the distribution function. The amplitude is obviously related to the maximum of the experimental distribution function. The ion temperature indicated in Figs. 7-9 is the result of the fit. In the three cases, the global agreement is good; all the experimental distribution functions, even those inside the sheath, are qualitatively well fitted by the model. The results seem to 


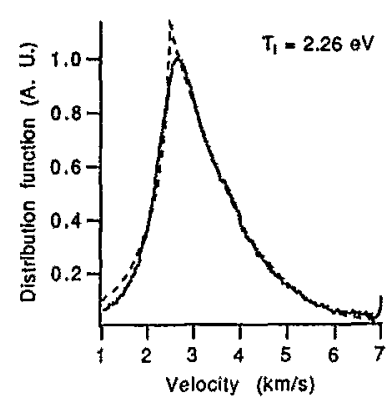

$x=1 \mathrm{~mm}$
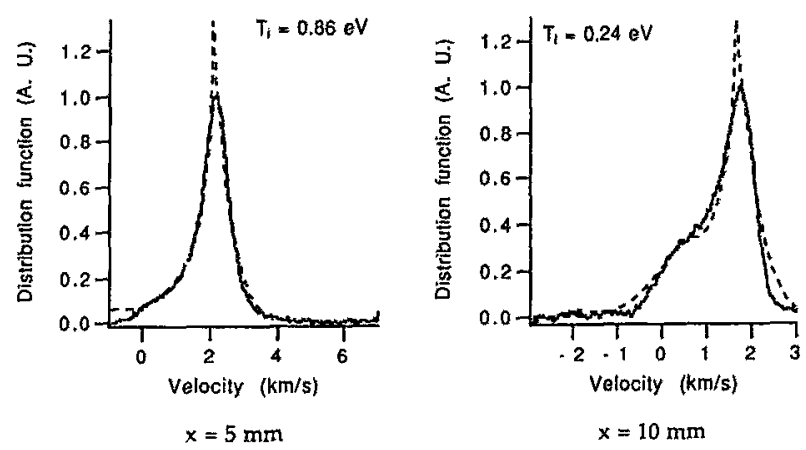

FIG. 7. Experimental (continuous line) and Emmert fit (dotted line) distribution functions with the endplates electrically floating and a pressure of $6 \times 10^{-4}$ Torr
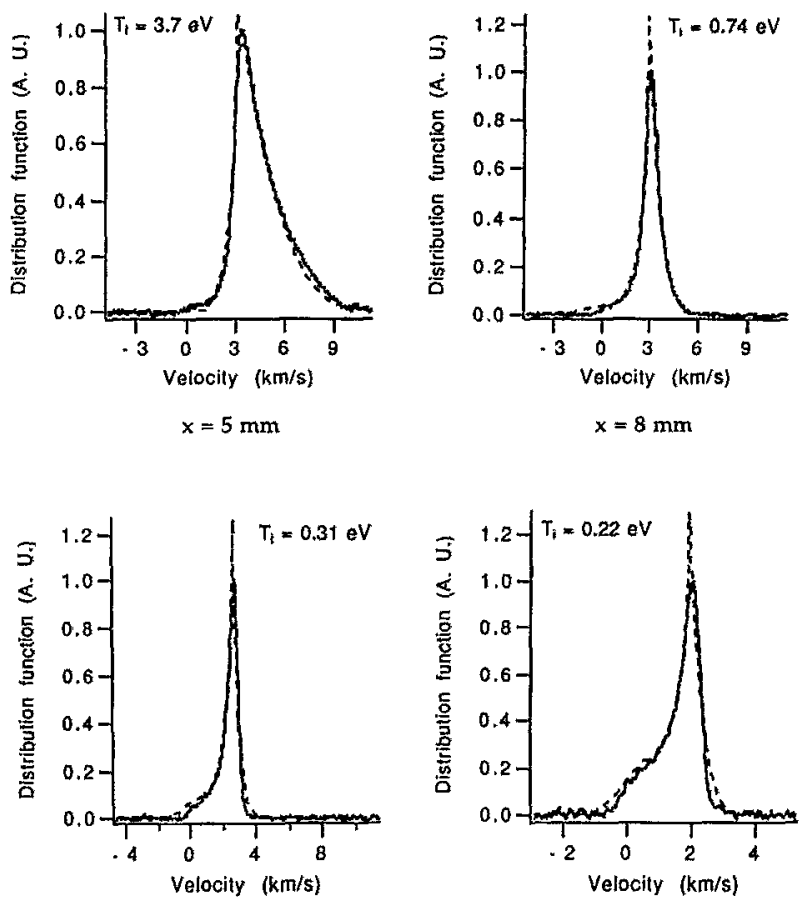

$x=10 \mathrm{~mm}$

FIG. 8. Experimental (continuous line) and Emmert fit (dotted line) distribution functions with the endplates electrically floating and a pressure of $10^{-4}$ Torr.
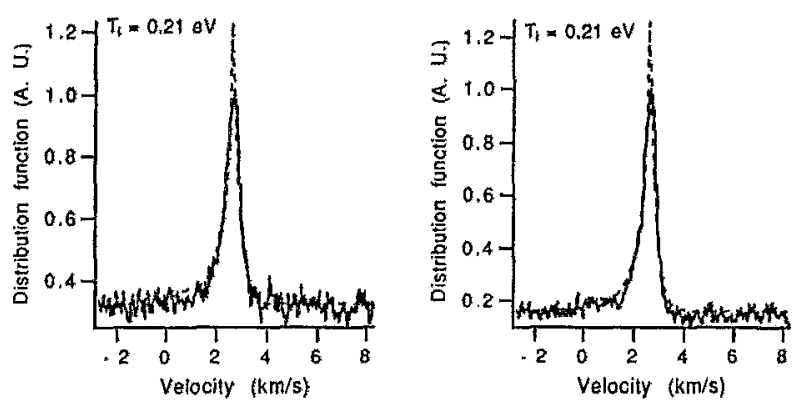

$\mathrm{x}=2 \mathrm{~mm}$

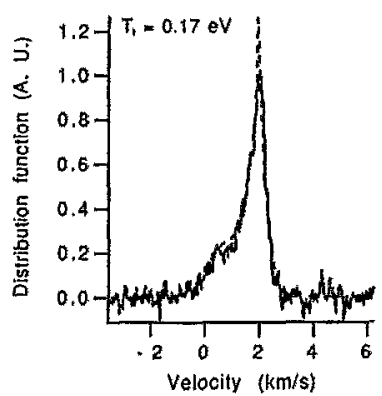

$x=15 \mathrm{~mm}$

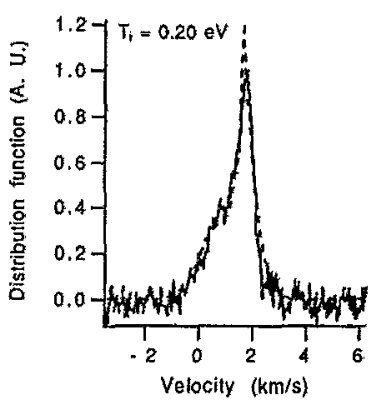

$x=20 \mathrm{~mm}$

FIG. 9. Experimental (continuous line) and Emmert fit (dotted line) distribution functions with the endplates grounded and a pressure of $10^{-4}$ Torr.

confirm the validity of Emmert et al. ${ }^{\prime}$ s model: ${ }^{13}$ The physical mechanism based on the energy of the created ions appears to be able to explain the ion dynamics.

Moreover, if the velocity distribution source function is taken to be Maxwellian as in Bissell's paper, ${ }^{21}$ the resulting distribution functions do not resemble the experimental ones: Especially in the plasma region where $\phi=0$, no hump appears on the distribution functions, contrary to our results.

Nevertheless, the ion temperature enhancement which is clearly observed near and inside the sheath is probably due to a mechanism which is missing in Emmert et al.'s theory. ${ }^{13}$ We noticed that this heating is anisotropic, since no increase in the ion temperature is observed in the transverse direction.

We first thought that this ion temperature enhancement was the result of an artifact due to the diagnostic. The spatial resolution in the radial direction is $2 \mathrm{~mm}$. This means that in a location where there is a velocity gradient, as in our case, the recorded velocity distribution function is in fact convoluted by an apparatus function. We have repeated our measurements with a slit shaped diaphragm which gives in the laser direction a spatial resolution of $0.2 \mathrm{~mm}$ and have observed the same temperature enhancement. Moreover, we have introduced a convolution by a rectangular function representing the effects of the finite spectral resolution of the diagnostic in the numerical program which fits the parameters of the model to the experimental data. For realistic width of the apparatus function, we still observe a temperature enhancement.

It is well known ${ }^{26}$ that the plasma obtained in a multipolar thermoelectronic discharge can exhibit two electron population with different temperatures. We verified it in our 


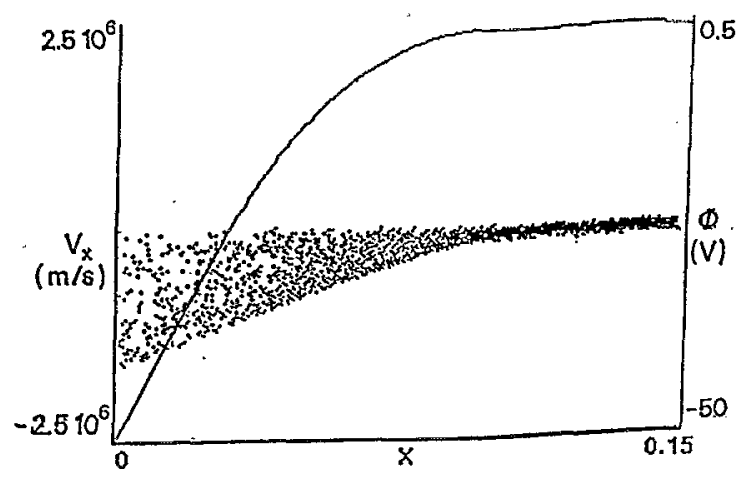

(a)

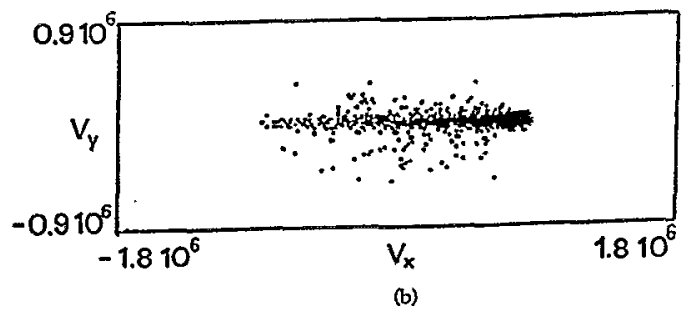

FIG. 10. (a) Ion phase space (dots) and potential $\Phi$ (continuous line) from the Birdsall's simulation PIC code. There is a large dispersion of the ion velocity $v_{x}$ in the region of potential gradient. (b) The dispersion of the velocity is anisotropic, in the direction of the electric field along $x$.

device: The semilog plot of a Langmuir probe characteristic curve is approximated by two straight lines which gives two electron temperatures, 1.75 and $11.3 \mathrm{eV}$ in the case of Fig. 7. Keeping the same assumptions as Emmert's ${ }^{13}$ except for the electron density, which we assumed to be given by a sum of two Boltzmann distributions with two different temperatures, we have deduced the ion distribution function again. The analytical expression obtained for the distribution function is very close to Emmert's one ${ }^{13}$ and there is no effect on the ion temperature. Only the constant coefficients and the argument of the Dawson function are different and this cannot explain the observed ion heating. We did not try to fit our experimental data with this new expression because it involves to many parameters.

It is important to notice that because of a low ionization rate and a large ion neutral charge exchange cross section for argon, the ion mean free path is relatively small $(12.5 \mathrm{~cm}$ for $6 \times 10^{-4}$ Torr) compared with the dimension of our device and the plasma is not really collisionless. In the region close to the sheath, where there is an important electric field, ion collisions with neutrals result in an anisotropic spreading of the thermal ion velocity. It can be easily seen with Birdsall's Plasma Device Particle-In-Cell code ${ }^{27,28}$ used to simulate a thermoionic diode where the plasma is created by ionizing collisions between a neutral gas and electrons emitted with a given velocity distribution function at $x=0$. Figure 10(a) shows the dependence of the longitudinal ion velocity spreading with the electric field. Figure 10(b) shows a strong anisotropic heating along the direction of the electric field.
This can explain the experimentally observed anisotropy in temperature.

This heating can also result from sheath fluctuations or from some more sophisticated mechanism that needs to be carefully characterized by other measurements.

\section{CONCLUSION}

We have presented the results of two different studies showing the influence of the boundary conditions on a low density $\left(10^{9} \mathrm{p} / \mathrm{cm}^{3}\right)$, low ionization rate argon plasma created by a thermoelectronic discharge in a multipolar device.

The first one shows a fast ion flux parallel to the magnets creating the multipolar magnetic field, which can be attributed to an $E \times B$ drift. Leakage aperture in the magnetic arches is found to be approximately the hybrid radius in previous experiments. We also observed ion leaks at the cusps, on the magnets.

The second experiment carried out without the multipolar magnetic field of the doors gives an insight on the plasma creation. The analytical one-dimension Emmert et al.'s kinetic mode ${ }^{13}$ seems able to explain qualitatively our results and the choice in this model of a flux-type source function seems entirely justified experimentally. However, there is experimental evidence for anisotropic ion heating, confirmed by a numerical simulation. It appears from the quantitative disagreement between theoretical previsions and experiments that collisional ion heating mechanism present in our device, like the charge exchange mechanism, has to be taken into account in the theory.

\section{ACKNOWLEDGMENTS}

The authors would like to thank J. Derouard and R. Stern for numerous advice and helpful discussions. The technical assistance of A. Totin, J. C. Chezeaux, and B. Squizzaro is greatly appreciated.

L. C. was supported by a grant from the Research and Technology Ministry.

'R. Measures, J. Appl. Phys. 39, 5232 (1968).

${ }^{2}$ R. A. Stern and J. A. Johnson, Phys. Rev. Lett. 34, 1548 (1975).

${ }^{3}$ R. A. Stern, D. N. Hill, and N. Rynn, Phys. Rev. Lett. 47, 792 (1981).

${ }^{4}$ D. N. Hill, S. Fornaca, and M. G. Wickham, Rev. Sci. Instrum. 54, 309 (1983).

${ }^{5}$ G. Bachet, L. Chérigier, M. Carrère, F. Doveil, Phys. Fluids B 5, 3097 (1993).

${ }^{6}$ L. Chérigier, Ph.D. thesis, University of Provence, Marseille, 1994.

${ }^{7}$ R. Limpaecher and K. R. Mackenzie, Rev. Sci. Instrum. 44, 726 (1973).

${ }^{8}$ M. Benhassine, J. C. Bourdessol, C. Gauthereau, J. Godiot, G. Mathieussent, and J. L. Rauch, Rev. Phys. Appl. 19, 545 (1984).

${ }^{9} \mathrm{C}$. Gauthereau and G. Matthieussent, J. Phys. 45, 1113 (1984).

${ }^{10} \mathrm{C}$. Gauthereau and G. Matthieussent, Phys. Lett. A 121, 342 (1987).

${ }^{11}$ K. N. Leung, N. Hershkowitz, and K. R. MacKenzie, Phys. Fluids 19, 1045 (1976)

${ }^{12}$ J. L. Ferreira, IEEE Trans. Plasma Sci. PS-20, 988 (1992).

${ }^{13}$ G. A. Emmert, R. M. Wieland, A. T. Mense, and J. N. Davidson, Phys. Fluids 23, 903 (1980).

${ }^{14}$ L. Tonks and I. Langmuir, Phys. Rev. 34, 876 (1929).

${ }^{15} \mathrm{D}$. Bohm, The Characteristics of Electrical Discharges in Magnetic Fields (McGraw-Hill, New York, 1949).

${ }^{16}$ E. R. Harrison and W. B. Thompson, Proc. Phys. Soc. London 74, 145 (1959).

${ }^{17}$ L. S. Hall, Proc. Phys. Soc. London 80, 309 (1962).

${ }^{18}$ A. Caruso and A. Cavaliere, Nuovo Cimento 26, 1389 (1962) 
${ }^{19} \mathrm{P}, \mathrm{N} . \mathrm{Hu}$ and S. Ziering, Phys. Fluids 9, 2168 (1966).

${ }^{20}$ S. Kuhn, Plasma Phys. 23. 881 (1981).

${ }^{21}$ R. C. Bissell and P. C. Johnson, Phys. Fluids 30, 2264 (1987).

${ }^{22}$ R. J. Procassini, C. K. Birdsall, and E. C. Morse, Phys. Fluids B 2, 3191 (1990).

${ }^{23}$ L. A. Schwager and C. K. Birdsall, Phys. Fluids B 2, 1057 (1990).

${ }^{24}$ T. E. Sheridan and J. Goree, Phys. Fluids B 3, 2796 (1991).
${ }^{25}$ J. M. Grossmann, S, B. Swanekamp, and P. F. Ottinger, Phys, Fluids B 4, 4.4 (1992).

${ }^{20} \mathrm{~K}$. Uehara, K. Yatsu, S. Hagiwara, and S. Kojima, Jpn. J. Appl. Phys. 14, 1761 (1975).

${ }^{27}$ C. K. Birdsall and A. B. Langdon, Plasma Physics via Computer Simulation (Hilger, Bristol 1991).

${ }^{28}$ C. K, Birdsall, IEEE Trans. Plasma Sci. PS-19. 65 (1991). 